LBL --28222

DE90 009049

\title{
STABILITY OF HIGH-BRILLIANCE SYNCHROTRON RADIATION SOURCES*
}

\author{
S. CHATTOPADHYAY \\ Arcelerator \& Fusion Research Division \\ Lawrence Berkeley Laboratory \\ 1 Cyclotron Rasd \\ Berkeley, CA 94720
}

December 1989

\section{DASClamma}

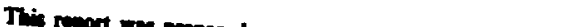

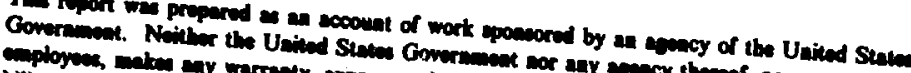

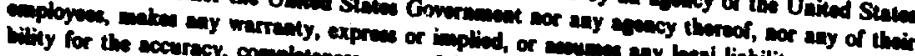

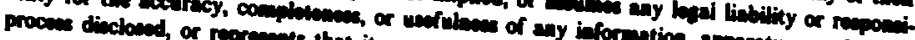

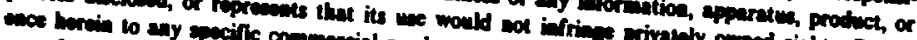

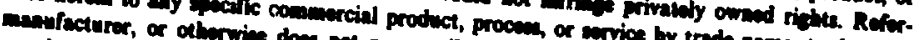

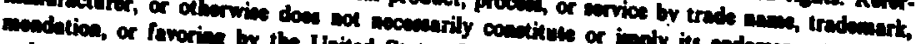

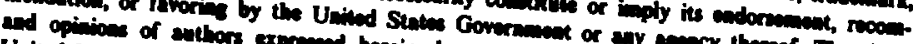

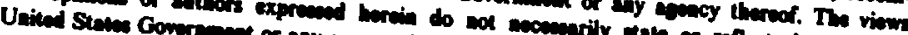

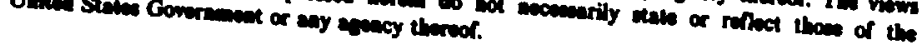

Invited talk presented at the 6th National Conference on Synchrotron Radiation Instrumentation, Berkeley, 1989; to be published in Nuclear Instruments \& Methods A.

* This work was supported by the Director, Office of Energy Research, Office of Basic Energy Sciences, Materials Sciences Division, of the U.S. Department of Energy under Contract No. DE-AC03-76SF00098. 
I.BL-28222

ESG Note-85

STABILITY OF HIGH-BRILLIANCE SYNCHROTRON RADIATION SOURCES*

\author{
S. CHATTOPADHYAY \\ Lawrence Berkeley Laboratory \\ 1 Cyclotron Road \\ Berkeley, CA 94720
}

\title{
1. Introduction
}

Driven by scientific interest in a variety of disciplines, there are several next generation synchrotron radiation sources being constructed or proposed worldwide. These are all based on large electron or positron storage rings. At present, there are two energy regimes being contemplated for these storage rings as dictated by scientific relevance - the 1-2 GeV range and the 6-8 GeV range. Projects in the former category are, for example, the Advanced Light Source (ALS) under construction at LBL, the Elettra project in Trieste, BESSY-II in Berlin, SRRC in Taiwan, the Pohang Light Source project at POS $;$ ECH in Korea, and LNLS in Brazil. Interestingly, storage rings usable as damping rings in linear colliders for high energy physics experiments and as drivers for ultraviolet free electron lasers also fall in this category from the point of view of their performance characteristics. Examples of projects in the 6-8 GeV category are the ESRF (Grenoble, France), the Advanced Photon Source at Argonne National Laboratory, Spring-8 project being built by RIKEN and JAERI in Japan, etc.

The requirements of all these storage rings are demanding and similar, namely to store stably an electron or positron beam of one hundred to a few hundred milliamperes of average current and transverse emittance less than or equal to $10 \pi \mathrm{nm}$-radian for a period of at least 5 to 10 hours. The common beam characteristics generic to all these rings are

* This work was supported by the Director, Office of Energy Research, Office of Basic Energy Sciences, Materials Sciences Division, of the U.S. Department of Energy under Contract No. DE-AC03-76SF00098. 
high current, low emittance, long lifetime and high stability. The combination of low emittance and high current gives the electron or positron beams exceptionally high brightness in phase space, an attribute necessary for the production of synchrotron radiation of high spectral brightress and flux. As beam dimensions decrease and intensities increase, the influence of many-body cooperative collective firer. mena on beam properties becomes more important and the beam becomes thare sensit. ' various noise sources as well. In addition, the beam behavior in most of these rlevices will be dominated by a number of periodic magnetic undulators or wigglers (known generically as insertion devices), which add complexities to the dynamics of beam orbits in storage rings. Such high brightness would be of little use unless it could be preserved via sophisticated diagnostics and control. Before we consider issues of beam stability, let us outline the typical characteristics and attributes of the photon source from the viewpoint of the scientific user.

\section{Characteristics of a Synchromon Radiation Sounce}

The photon source can be characterized as follows:

- Directional "ray" characteristics:

- Horizontal position, $x$.

- Horizontal angle, $x^{\prime}=\mathrm{dx} / \mathrm{ds}$.

- Vertical position, $y$.

- Vertical angle, $y^{\prime}=\mathrm{dy} / \mathrm{ds}$.

- Transverse profile $\left(\sigma_{\mathrm{x}}, \sigma_{\mathrm{y}}\right)$.

- Longitudinal (temporal) profile ( $\sigma_{\tau}$ or $\sigma_{2}$ and pulse structure).

- Polarization (special configuration of the electric and magnetic field vectors of the electromagnetic wave).

- Intensity (photon flux) and "spectral brilliance."

- Photon spectrum. 
Systematic and random perturbations. (noise or jitter) of any kind will affect all the above characteristics. Stability over time in particular is of special relevance for usability.

The effect of perturbations internal or external to the storage ring on the "ray" characteristics is obvious. The transverse profile is largely determined by the emittance $\left(\varepsilon_{x, y}\right)$ of the electron or positron beam and the liftice functions of the storage ring electromagnetic structure-beta functions $\beta_{x, y}(s)$ and dispersion $\eta_{x, y}(s)$. Moreover, intrabeam Coulomb collisions and interaction with residual gas will play roles in determining the beam's spatis distribution and its time evolution (lifetime). Coherent instabilities may limit the emittance and intensity of the beam and determine its temporal stability. These will manifest themselves directly in the photon flux and spectral brilliance. In addition, the nonlinear nature of the storage ring magnetic lattice, including undulators, will result in distortions of the electron beam is transverse phase space distribution, which will manifest themselves in the photon beam. Finally, the photon spectrum will be determined by the magnitude of the bending or undulator magnetic field, the energy of the beam, the $K$ value of the undulator $\left(\mathrm{K} \equiv 0.934 \mathrm{~B}_{[\text {Tesla] }} \lambda_{\mathrm{u}[\mathrm{cm} .]}\right)$ and the harmonic used. The mechanical and magnetic precision and stability of these magnetic systems are crucial to preserving the desired photon spectra. Finally, increasing brilliance necessarily brings with it the increasingly difficult task of achieving the required stability in ever smaller and brighter beams.

\section{Play of the Opposites}

Design of a low-emittance, high-brillance synchrotron radiation storage ring involves reconciling the conflicting requirements of the storage ring lattice, if systems, beam intensity and energy, etc. An optimum design requires a completely self-consistent configuration. Table 1 illustrates some of the conflicting demands imposed on the storage ring parameters. While beam stability becomes easier to assure with increasing beam energy, scientific justifications constrain the energy to a certain maximum value. The 
demand for high brilliance implies low emittance and high current, both of which degrade beam emittance via intra-beam roulomb scattering and decrease coherent stability via high phase-space density. There is competition even within these two effects - stability against microwave instability demanding a large momentum compaction factor $\alpha$ versus minimization of intra-beam scattering rate demanding a lower $\alpha$. To prevent loss of particles via large-angle Coulomb scattering (Touschek effect), one should provide a large voltage on the if cavities. However, this would imply more if cells, hence more cost (and susceptibility to coupled multibunch instability driven by the higher-order modes of the if cavities). Reducing the magnetic gap of undulators is desirable in order to make them stronger and to facilitute shorter period lengths, leading to higher-energy photons. Too small a gap, however, will impose aperiure restrictions upon the beam that are unacceptable from the point of view of beam lifetime and coherent stability. Similarly, space and experimental requirements may favor short-period undulators, which are undesirable from the nonlinear aspect of stable dynamic aperture of the storage ring. Finally, while microscopic interaction of the charged particle beam with the generated radiation is weaker for longer-wavelength of radiation, wavelength is constrained by design to be no longer than a limiting value dictated by experimental interests. 


\section{Table 1}

Play of the opposites

Left-hand column describes the storage ring and beam parameters. The column under the upward arrow describes considerations and factors often demanding higher values of the parameters to the left. The column under the downwand arrow describes those factors that often demand lower values of the same corresponding parameters.

\begin{tabular}{|c|c|c|}
\hline & $\Uparrow$ & $\Downarrow$ \\
\hline Energy, $\gamma$ & Beam stability & Scientific interests \\
\hline Emittance, $\varepsilon$ & Intrabeam scattering & Beam brightness \\
\hline Momentum spread, $\sigma_{\mathrm{p}}$ & Microwave instability & Beam brightness \\
\hline Momentum compaction, $\alpha$ & Microwave instability & Intrabeam scattering \\
\hline RF Voltage, $V_{R F}$ & Touschek lifetime & $\begin{array}{l}\text { Cost; coupled bunch } \\
\text { instability }\end{array}$ \\
\hline Undulator magnetic gap, $d$ & Beam lifetime; impedance & $\begin{array}{l}\text { Strong undulator, } \\
\text { high energy photons }\end{array}$ \\
\hline Undulator period, $\lambda_{\mathrm{u}}$ & $\begin{array}{l}\text { High undulator K parameter, } \\
\text { small nonlinearity }\end{array}$ & $\begin{array}{l}\text { Small undulator length; } \\
\text { experimental interests }\end{array}$ \\
\hline Radiation wavelength, $\lambda$ & $\begin{array}{l}\text { Beam-radiation interaction } \\
\text { weak; also implies large } \lambda_{u}\end{array}$ & $\begin{array}{l}\text { By design, since } \\
\text { experimental interests are } \\
\text { strong }\end{array}$ \\
\hline
\end{tabular}

\section{Stability}

Storage-ring bending magnets define an average ideal closed orbit for the electron beam. Spatial confinement is provided transversely by the focusing and defocusing quadrupole magnetic lenses and longitudinally by the rf cavity fields oscillating sinusoidally in time. These elements create a potential well in which particles execute stable bounded oscillations with small amplitudes. In a perfect situation, the ideal closed orbit passes 
through the magnetic centers (magnetic field $=0$ ) of the quadrupoles and slightly off the electrical oscillation center (longitudinal electric field $=0$ ) of the RF cavities (to make up for the synchrotron radiation energy loss). Particles with $x, x^{\prime}(\equiv d x / d s), y, y^{\prime}(\equiv d y / d s), z$, $\mathrm{dz} / \mathrm{dt}$ deviating from the ideal closed orbit execute betatron and synchrotron oscillations around that orbit. All oscillations are bounded transversely within an envelope $\sqrt{\varepsilon_{x, y} \beta_{x, y}(s)}$ which has different amplitudes along the azimuth s of the ideal closed orbit, $\varepsilon_{x, y}$ is the invariant emittance and $\beta_{x, y}(s)$ are lattice amplinude or beta functions.

The actual closed orbit in a real machine deviates from the ideally desired closed orbit, due to inevitable errors in survey and alignment. Typically, maximum value of this deviation could range from millimeters to a centimeter a few arising from a realistically achievable survey and alignment error of $0.1 \mathrm{~mm}$. This is a time-independent (stationary) configuration and can be improved by a closed orbit measurement and correction scheme, employed in all modern storage rings. An irreducible residue of $0.1 \mathrm{~mm}$ to $0.5 \mathrm{~mm}$ in maximum closed orbit deviation from ideality is achievable after a convergent series of iterations.

Life would be easier in this static situation if everything were independent of time and oscillations were linear even if particles were pumped to large amplitudes. The challenge of control of the photon source stems from the reality of time-dependent perturbations and the essential nonlinearity of the beam dynamics at large amplitudes. There are always long term ground motions and various vibrations and noise sources at faster time scales. Particles are also subjected to large oscillation amplitudes at injection as well as continually during the beam's lifetime by various scattering processes. In addition there are other time-dependent processes, such as coherent beam instabilities, oscillations of trapped ions interacting with the beam, etc. The frequencies and time scales of these various processes, their sources, manifestations in the beam and ring properties, monitoring systems and possible cures can be grasped by a look at Fig. 1. 


\section{Obit Control}

Issues that are usually addressed in orbit control are identification of various noise sources, definition of stability requirements in $\left(x, x^{\prime}, y, y^{\prime}\right)$ and determination of requirements and design of proper feedback systems, taking into account photon beam monitors, frequency responses, cross-talk between various feedback loops, etc.

The noise sources include: ground motion, temperature change of cooling water or ambient air, external power source variations, various power supplies (especially those for the booster), orbit bump "leakage", correction magnet hysteresis, beam position monitor stability, insertion device gap changes during scanning, ion trapping, and thermal effects on mirrors or monochromators, vibration of monochromators and various other 'cultural' noise.

As an example of stability requirement, one may require the stability to be such as to allow less then $1 \%$ variation in throughput beyond a properly placed slit. Let us say that this translates into a ertical position error $\Delta_{y}$ of about $17 \%$ of the beam size $\sigma_{y}$. For $\sigma_{y}=40 \mu$ then, the requirement is $\Delta_{y}=6 \mu$.

Typically, photon-to-electron control loop feedback is achieved by detecting the photon beam at two synchrotron light beam monitors, extracting position and angle errors $\Delta y, \Delta y^{\prime}$ and feeding properly processed and amplified information through separate $y$ and $y^{\prime}$ loops to steering magnets on both sides of an undulator, say.

A typical orbit feedback loop is shown in Fig. 2. Here $\vec{y}=\left(y, y^{\prime}\right)$ is the beam position at the center of the insertion device, $K_{D} \equiv\left(y, y^{\prime}\right)$ and is given by the photon beam monitor and, $R$ = the requested $\left(y, y^{\prime}\right) G(s)$ is the control loop compensation matrix including vacuum chamber response and transfer function, $K_{0}$ is the closed orbit beam response at the insertion device center, and $u \equiv \Delta\left(y, y^{\prime}\right)$ at the center of the insertion device due to "noise." 
The "position" and "angle" bumps produced by the steering magnets in the two loops are illustrated in Fig. 3.

Typical instruments and hardware for orbit control are photon beam monitors, electron beam position monitors, correcting magnets and electronics of the feedback system. Feedback modeling requires proper description of Loop Transfer Functions (including vacuum chamber magnetic penetration) and of cross-talk in multiple-loop systems that control more than one photon beam.

\section{Nonlinenr Dynamic Stability}

The necessary properties for an optimized low emittance storage ring are small beam size and angular divergence. One requires a strong focusing lattice with low beta and low dispersion, requiring strong quadrupoles and strong sextupoles as well to compensate for the generated chromaticity. Sextupoles introduce nonlinearity into the orbit dynamics. In addition undulators perturb the linear optics and further add to the nonlinear effects. The result of all this is typically a small dynamic aperture for the electron beam - the aperture in transverse space beyond which an electron is dynamically unstable and cannot be contained by the accelerator's magnetic structure.

The calculated effect of magnet imperfections, nonlinear sextupole fields and undulators for the Advanced Light Source under construction at LBL is shown in Fig. 4. 1 The aperture, although small, is still larger than the physical aperture available. Care has to be exercised in the design phase to ensure adequate aperture. In principle, if there are enough independently powered quadrupoles, the effect of many undulators can be linearly compensated by proper quadrupole settings. Flexibility and room for tuning has to be kept in mind in detailed design considerations. Large amplitude beam motion is expected at injection due to injoction errors. Besides, particles continually scatter off to large amplitudes by various intrabeam and beam-gas collisions. 


\section{Coherent Stability and Control}

A charged particle beam interacts with itself via its Coulomb space charge as well as via image charges and currents in the surrounding vacuum chamber walls and discontinuities. The self-interaction of these bunches via radiation into the surrounding electromagnetic structures is characterized by the "beam coupling impedances". These latter provide a measure of the self-induced longitudinal voltages, $V(\omega)$ (or transversely deflecting fields) in response to modulations of the longitudinal beam current, $I(\omega)$ (or transverse motion), at a frequency $\omega=2 \pi f$ :

$$
V(\omega)=-Z(\omega) \cdot I(\omega),
$$

where $Z(\omega)$ is the longitudinal beam coupling impedance. Under certain circumstances, microscopic or macroscopic coherent motions of the bunch can self-sustain and regenerate via proper phase relationships. Such oscillations can be stable or may grow depending on the impedance. Simply stated, the frequency of coherent motion is shifted from the frequency of incoherent orbit motion and generated by the impedance $Z(\omega)$ acconding to straightforward first-order perturbation theory as:

$$
\Delta \omega_{\text {coherent }} \propto \text { i } \frac{\left\langle\rho_{0}(\omega)\left|\frac{Z(\omega)}{\omega}\right| \rho_{0}(\omega)\right\rangle}{\rho_{0}(\omega)\left|\rho_{0}(\omega)\right\rangle} \text {, }
$$

where $p_{0}(\omega)$ is the unperturbed stationary density eigenspectrum of the bunch and $\langle\ldots . . . . .$. implies weighted integrations over $\omega$. The impedance $Z(\omega)$ is a complex quantity in general, having both real (resistive) and imaginary (reactive) parts. If $\Delta \omega_{\text {coh }}$ has an imaginary par,, coherent oscillations may grow (or decay) leading to an instability.

A typical impedance spectrum seen by a beam up to extremely high frequencies is shown schematically in Fig. 5 in a plot of its real part vs. frequency. At low frequencies there are a series of sharp resonances associated with higher-order electromagnetic modes of the rf cells and any other cavity like elements in the beam chamber that can trap 
electromagnetic energy. These continue upto the beam-tube wave guide cutoff frequency, beyond which impedance falls off according to a certain scaling law, depending on the interplay of constructive and destructive interference of diffracted waves from various discontinuities in the beam chamber. At even higher frequencies, the process of synchrotron radiation itself induces coberence within a bunch and generates selfimpedance. Eventually the impedance falls to negligible values beyond a frequency of $\omega=$ $\gamma /$ a corresponding to the diffraction-limited regime sturting at $\lambda=\alpha \gamma$, where a being the typical beam chamber dimension and $\gamma$ is the relativistic gamma factor.

Careful policing of the impedance budget of the storage ring at the design and construction phase and active devection and if feedback of the coherent beam motion are essential to controlling these instabilities.

\section{Summary}

There are certain important generic features of high brightness, low emittance storage rings for synchrotron radiation that require special attention. We stress the following:

- Precise control of orbit stability affected by various noise sources. Careful design of photon-to-electron feedback systems, minimizing crosstalk, is essential.

- Amplitude limitation due to nonlinearity leading to reduced dynamic aperture and Touschei beam lifetime.

- Minimizing high frequency impedance - instability dre to short bunch length.

- Special attention to higher-order-mode damping of if cavities and strong if feedback systems, to control coupled multibunch instabilities.

- Effects of ions - the cure is a large gap in the train of bunches and/or the use of clearing electrodes, or the use of positrons rather than elsctrons.

- Inclusion of Insertion Devices (undulators) as an integral part of the ring at the design stage whenever possible. 
Acknowlederment

I am grateful to members of the Exploratory Studies Group at LBL working on the ALS project for educating me through their work on the topics covered in this article.

\section{Reference:}

1. A. Jackson, E. Forest, H. Nishimura and M.S. Zisman, "The Effects of Insertion Devices on Beam Dynamics in the ALS," LBL-25958, Proceedings of the IEEE Particle Accelerator Conference, Chicago, II, March 20-23, 1989. 


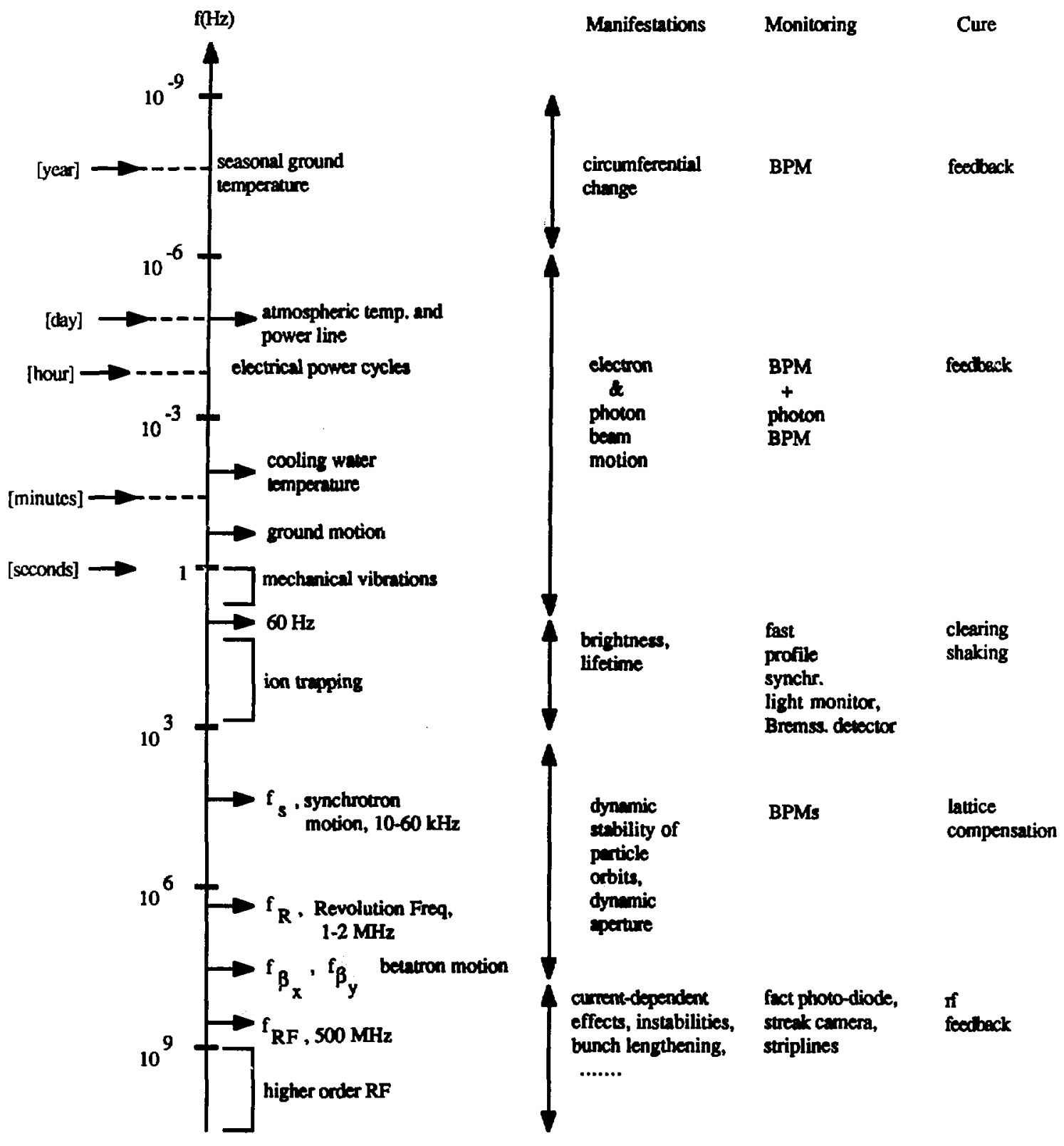

Fig. 1 Various frequencies and time scales 


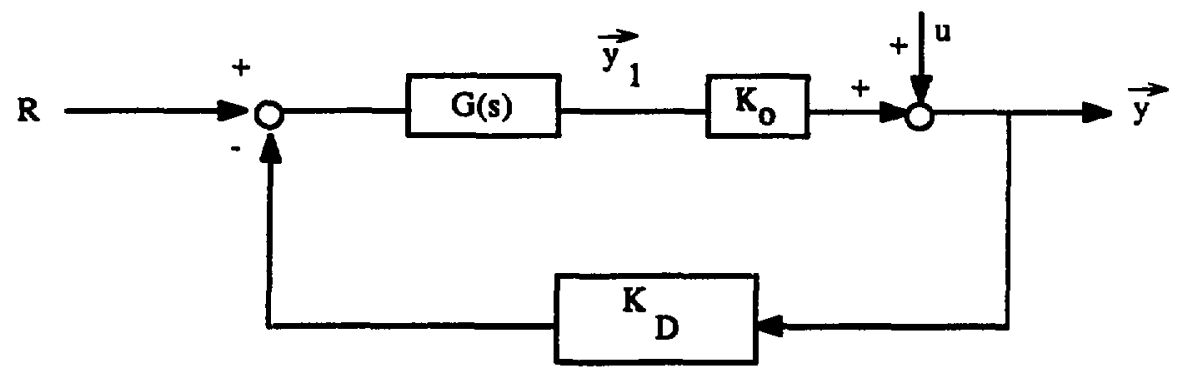

Fig. 2 Typical orbit feedback loop
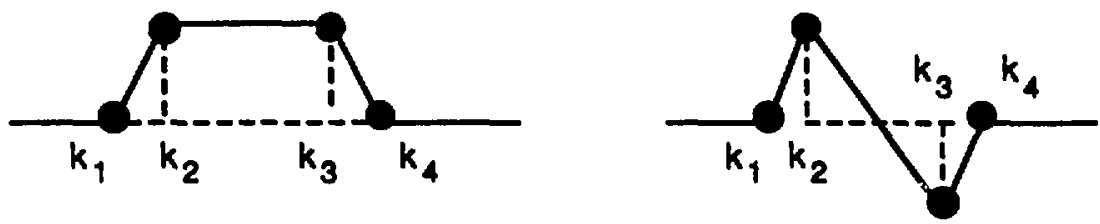

" Position " bump

" Angle " bump

Fig. 3 


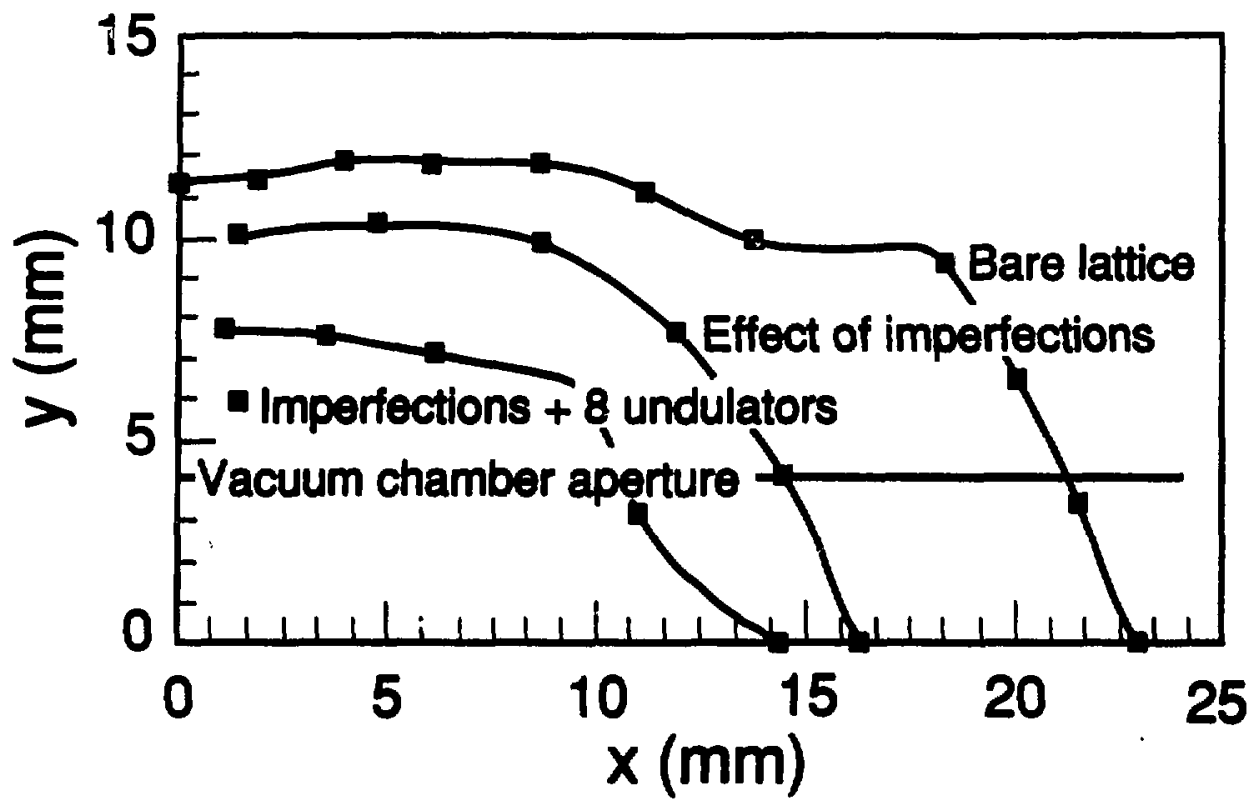

XEL $901-6701$

Fig. 4 Effect of Magnet Imperfections and Undulators on the Dynamic Aperture of the Advanced Light Source. 


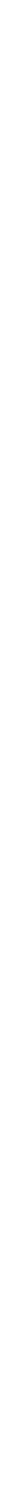

X:4 8912-7343

Fig. 5 Typical impedance spectrum (real part) seen by a storage ring beam up to extremely high frequencies. 


\section{DSCLAIMER}

This document we propened an an scoount of wort spowored by the United Stuks Government. Neither the Uniwd Stmas

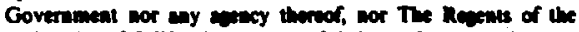

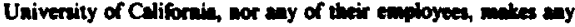

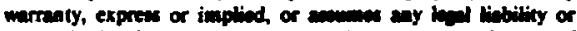

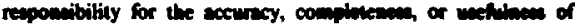

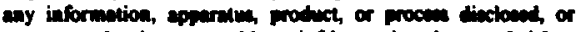

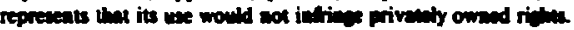

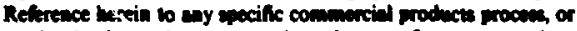

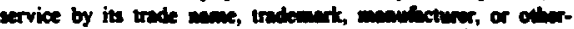
wise, does wot neceserity cometitule or insty its endorsoment, recommeadetion, or hvoring by the Ulind Swme Government or any anacy thereof, or The hemats of the Univensity of Californic. The view and opinion of anbors expresud bertin do

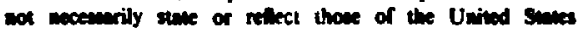
Government or any enewcy therwof or The Renewts of the Univerity of Celiforia end shall not be wad for edverticing or product endorsement puposet.

Lewrence Benteley Laboreliony in en equel opportunity employer. 